\title{
ArcheoSciences
}

Revue d'archéométrie

\section{La tombe mycénienne de Kazanaki (Volos) et le mythe de la Toison d'or}

The Mycenaean tomb of Kazanaki (Volos) and the myth of the Golden Fleece

Vassiliki Adrimi-Sismani, Maria Filomena Guerra and Philippe Walter

\section{(2) OpenEdition}

\section{Electronic version}

URL: https://journals.openedition.org/archeosciences/2121

DOI: 10.4000/archeosciences. 2121

ISBN: 978-2-7535-1598-7

ISSN: 2104-3728

Publisher

Presses universitaires de Rennes

\section{Printed version}

Date of publication: 31 December 2009

ISBN: 978-2-7535-1181-1

ISSN: $1960-1360$

\section{Electronic reference}

Vassiliki Adrimi-Sismani, Maria Filomena Guerra and Philippe Walter, "La tombe mycénienne de Kazanaki (Volos) et le mythe de la Toison d'or", ArcheoSciences [Online], 33 | 2009, Online since 10 December 2012, connection on 28 January 2022. URL: http://journals.openedition.org/ archeosciences/2121; DOI: https://doi.org/10.4000/archeosciences.2121 


\title{
La tombe mycénienne de Kazanaki (Volos) et le mythe de la Toison d'or
}

\author{
The Mycenaean tomb of Kazanaki (Volos) and the myth of the Golden Fleece
}

\author{
Vassiliki Adrimi-Sismani*, Maria Filomena Guerra** et Philippe Walter**
}

Résumé : Parmi les sites mycéniens de Thessalie, Dimini, situé près de Volos, pourrait correspondre au fameux centre mycénien d’Iolkos, ville gouvernée par Pélias et qui est à l'origine du mythe de Jason et de la Toison d'or, célèbre peau de bélier reliée à la récupération d'or des alluvions. Une nouvelle tombe mycénienne à tholos, datée de 1350 av. J.-C., découverte en 2004 à Kazanaki, a livré un nombre très important d'objets en or. L'examen et l'analyse élémentaire de feuilles et disques d'or, colliers et perles de collier au LC2RMF et à Volos avec des équipements portables, nous a permis de faire une première approche des techniques orfèvres de la période mycénienne et de mettre en évidence l'utilisation d'or d'origine alluvionnaire, parfois avec ajout de cuivre, pour la fabrication de l'orfèvrerie.

\begin{abstract}
Among the Mycenaean sites in Thessaly, Dimini, situated close to Volos, could correspond to the famous Mycenaean centre of Iolkos, ruled by Pellias, the site that was at the origin of the myth of Jason and the Golden Fleece, the famed ram's skin related to the exploitation of alluvial gold. A new Mycenaean tholos tomb discovered in Kazanaki in 2004, and dated to 1350 BC, brought to light a very important number of gold objects. The examination and analysis of gold foils and discs, necklaces, and necklace beads have been undertaken at the LC2RMF laboratory and at Volos with portable systems. In this contribution, we present a first approach to the Mycenaean goldsmithing techniques and we demonstrate that the goldwork was produced with alluvial gold, sometimes with the addition of copper.
\end{abstract}

Mots-clés : Mycènes, Toison d'or, analyse, orfèvrerie, Kazanaki.

Keywords: Mycenae, Golden Fleece, analysis, goldwork, Kazanaki.

\section{INTRODUCTION}

Une tradition mythologique très riche relie la Thessalie à la civilisation mycénienne, à la famille royale d'Iolkos (AdrimiSismani, 2007) et au mythe de Jason et des Argonautes, envoyés en Colchide - la région du Caucase en mer Noire pour chercher la Toison d'or.

Environ 200 sites mycéniens ont pu être identifiés en Thessalie, parmi lesquels Dimini, situé près du port de la ville moderne de Volos (Adrimi-Sismani, 2004), qui comporte des ateliers d'orfèvrerie, avec des outils en bronze, et d'importants entrepôts (Adrimi-Sismani et Godart, 2005). Ces espaces administratifs, économiques et religieux avec les habitats voisins de Palia/Kastro Volos et Pefkakia et quatre tombes à tholos destinées à l'enterrement de membres de familles royales, semblent correspondre au fameux centre d'Iolkos.

* Archaeological Institut of Thessalian Studies-74-76 Gambetta Str., 38221 Volos Greece. (aiths@culture.gr)

** Laboratoire du Centre de Recherche et de Restauration des Musées de France, UMR 171 CNRS - 14, quai François-Mitterrand, 75001 Paris, France (maria.guerra@culture.gouv.fr), (philippe.walter@culture.fr) 
Pendant les travaux effectués dans les environs nord de la ville moderne de Volos une quatrième tombe à tholos, de la période HR IIIA2 - HR IIIB1 (1350 av. J.-C.), a été mise à jour à Kazanaki, en 2004 (Adrimi-Sismani, 2008). La tombe comporte un long dromos, une entrée et une chambre funéraire circulaire de 6,7 $\mathrm{m}$ de diamètre, avec quatre tombes à fosse. Des signes incisés ont été repérés sur le linteau supérieur de la façade monumentale de la tombe. Des ossements de plusieurs corps ont été identifiés (Papanastasiou, 2006). Tous les objets, parmi lesquels un nombre important en or, et les ossements avaient été regroupés dans un seul tas, brûlés in situ et puis remis dans les tombes à fosse, qui ont été ensuite scellées.

Pour approcher la question de l'exploitation de gisements d'or alluvionnaire, associés au mythe des Argonautes et de la Toison d'or, 17 feuilles et disques de la tombe de Kazanaki ont été déplacés au C2RMF pour être analysés à l'accélérateur AGLAE. D'autres éléments en or ont été étudiés in situ avec des équipements portables, pour faire une première approche aux techniques orfèvres utilisées dans la production d'objets mycéniens.

\section{Méthodes}

Les objets sélectionnés pour ce travail ont été examinés sous loupe binoculaire ainsi que, au C2RMF, par radiographie $\mathrm{X}$ et par microscopie électronique à balayage (MEB) avec un Philips XL30 ESEM en mode SE à $20 \mathrm{kV}$. Leur analyse élémentaire a été effectuée à Volos avec un dispositif d'analyse par spectroscopie de fluorescence des rayons $\mathrm{X}$ basé sur un tube à rayons $\mathrm{X}$ Moxtek à anode fine d'argent et fonctionnant à $35 \mathrm{kV}$ et $95 \mu \mathrm{A}$. Le détecteur est une diode SDD AXAS-V de Ketek, refroidie par effet Peltier. Sa résolution en énergie est de $140 \mathrm{eV}$ à $5.9 \mathrm{keV}$ dans les conditions usuelles de fonctionnement. La quantification des données est obtenue avec le logiciel PyMCA (Sole et al., 2007) par la méthode des paramètres fondamentaux, après modélisation précise de la source de rayons $\mathrm{X}$. Les résultats quantitatifs ont été validés dans différents cas (Gianoncelli et al., 2006; Viguerie et al., 2009) et pour les ors, une série de standards ont permis de vérifier la justesse des résultats (Tableau 1).

Au C2RMF l'analyse élémentaire est réalisée à l'accélérateur AGLAE au moyen des techniques PIXE et PIXE-XRF (particle induced X-ray emission et FX induite par PIXE) avec un faisceau de protons de $3 \mathrm{MeV}$ extrait à l'air. La technique PIXE utilise deux détecteurs de $\mathrm{Si}(\mathrm{Li})$, un dédié à la détection des éléments majeurs et l'autre, avec un filtre sélectif de $75 \mu \mathrm{m}$ de $\mathrm{Cu}$, dédié à la mesure des éléments mineurs et traces (Guerra et Calligaro, 2004). La technique

\begin{tabular}{|c|c|c|c|c|}
\hline & & $\mathrm{Au} \%$ & Ag \% & $\mathrm{Cu} \%$ \\
\hline \multirow{2}{*}{ Lyon Allemand 6917} & \multirow{2}{*}{$\begin{array}{l}\text { mesuré } \\
\text { certifié }\end{array}$} & 74,2 & 18,3 & 7,5 \\
\hline & & 76,0 & 17,0 & 7,0 \\
\hline \multirow{2}{*}{ Lyon Allemand A } & \multirow{2}{*}{$\begin{array}{l}\text { mesuré } \\
\text { certifié }\end{array}$} & 90,1 & 5,3 & 4,6 \\
\hline & & 92,0 & 4,0 & 4,0 \\
\hline \multirow{2}{*}{ Lyon Allemand 893} & \multirow{2}{*}{$\begin{array}{l}\text { mesuré } \\
\text { certifié }\end{array}$} & 72,6 & 7,4 & 20,0 \\
\hline & & 75,2 & 5,7 & 19,1 \\
\hline \multirow{2}{*}{ Authentico E-2 } & \multirow{2}{*}{$\begin{array}{l}\text { mesuré } \\
\text { certifié }\end{array}$} & 56,7 & 42,8 & 0,5 \\
\hline & & 56,0 & 43,5 & 0,5 \\
\hline \multirow{2}{*}{ Authentico A-1 } & \multirow{2}{*}{$\begin{array}{l}\text { mesuré } \\
\text { certifié }\end{array}$} & 91,0 & 7,7 & 1,3 \\
\hline & & 92,6 & 6,4 & 1,0 \\
\hline \multirow{2}{*}{ Authentico A-2 } & \multirow{2}{*}{$\begin{array}{l}\text { mesuré } \\
\text { certifié }\end{array}$} & 73,8 & 23,4 & 2,8 \\
\hline & & 74,6 & 22,6 & 2,8 \\
\hline \multirow[t]{2}{*}{ Authentico E-1 } & \multirow{2}{*}{$\begin{array}{l}\text { mesuré } \\
\text { certifié }\end{array}$} & 53,1 & 46,8 & 0,1 \\
\hline & & 50,8 & 49,2 & 0,0 \\
\hline
\end{tabular}

Tableau 1 : Valeurs certifiées et mesurées par fluorescence à rayons $\mathrm{X}$ d'un groupe de standards.

Table 1: Theoretical values and compositional results for a group of standards analysed by XRF.

PIXE-XRF utilise pour la mesure du Pt dans les alliages en or, une cible primaire de As et un filtre de $25 \mu \mathrm{m}$ de $\mathrm{Zn}$ (Guerra et al., 2005; Guerra, 2004).

\section{Résultats et Discussion}

Nous avons étudié 22 fragments de bandes d'or décorées en ondulé et d'épaisseur et tailles différentes, 14 disques, 13 perles de collier à doubles spirales et à papyrus, et 2 colliers à rosettes et fleurs de lis (types de perles selon Higgins, 1980). La composition élémentaire des objets se trouve sur le Tableau 2.

\section{Les feuilles et les disques}

Les feuilles et les disques sont fabriqués par martelage, des orifices de 130-140 $\mu \mathrm{m}$ de diamètre permettent leur application sur des vêtements (Higgins, 1980). Leur morphologie, l'absence de traces d'usure et leur fine épaisseur indiquent qu'il s'agit d'une production à but funéraire. Certaines feuilles, très fines et froissées, ne révèlent leur décoration ondulée qu'à la radiographie X. Leur composition est homogène (Tableau 2), seules deux feuilles possèdent des teneurs en $\mathrm{Cu}$ supérieures à $2 \%$, et correspond à l'électrum naturel (selon la définition de Pline, Zehnacker, 1983). D'autres objets possèdent des teneurs identiques, comme une épingle à cheveux du III ${ }^{e}$ millénaire, de typologie proche de l'épingle de Troie (Swann et al., 1997). 


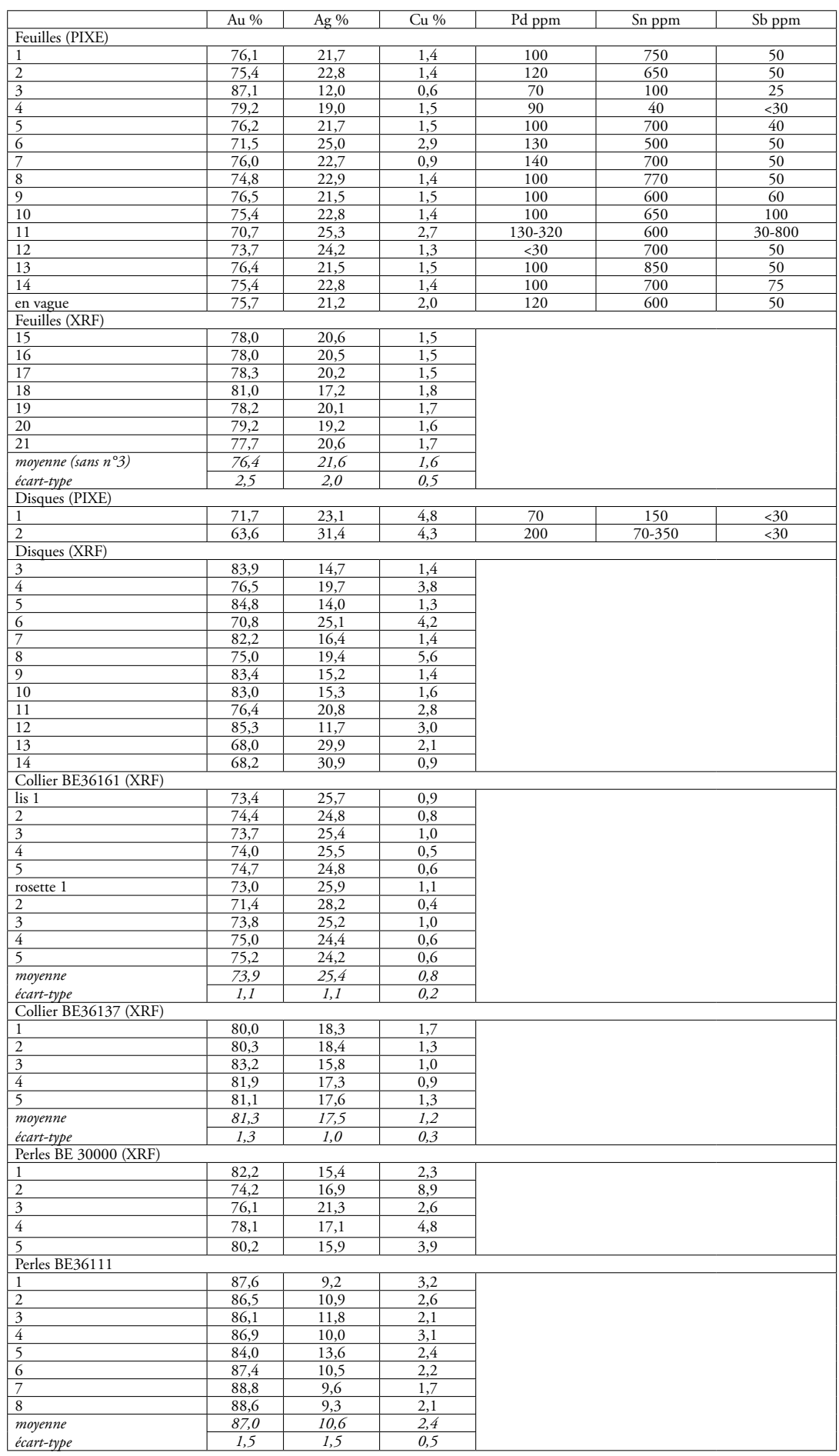

Tableau 2 : Résultats obtenus par PIXE et FX portable pour tous les objets de Kazanaki analysés et pour des objets mycéniens analysés par d'autres auteurs.

Table 2: Compositional results for all the analysed objects from Kazanaki by XRF and PIXE and for a few Mycenaean objects analysed by other authors.

\begin{tabular}{|l|l|c|c|c|}
\hline \multicolumn{2}{|l|}{ Autres auteurs } & $\mathrm{Au} \%$ & $\mathrm{Ag} \%$ & $\mathrm{Cu} \%$ \\
\hline \multirow{2}{*}{ Schliemann 1878} & Tombe 4 & 89,36 & 8,55 & 0,57 \\
\cline { 2 - 5 } & Tombe 4 & 73,11 & 23,37 & 2,22 \\
\hline \multirow{2}{*}{ Hackens 1993} & coupe moyenne & 82,45 & 17,47 & 0,08 \\
\cline { 2 - 5 } & écart-type & 0,78 & 0,76 & 0,04 \\
\hline \multirow{2}{*}{ Hartmann 1970} & bande & 87,85 & 12,17 & \\
\cline { 2 - 5 } & diadème & 75,1 & 24,9 & 0,5 \\
\hline \multirow{3}{*}{$\begin{array}{l}\text { Vavelidis and Andreou } \\
2008\end{array}$} & feuille & 90,3 & 9,25 & 0,55 \\
\cline { 2 - 5 } & perle & 77,5 & 22 & $<0,10$ \\
\cline { 2 - 5 } & fil & 99,8 & 0,15 & 3,5 \\
\cline { 2 - 5 } & globule & 78,8 & 20,35 & \\
\hline
\end{tabular}



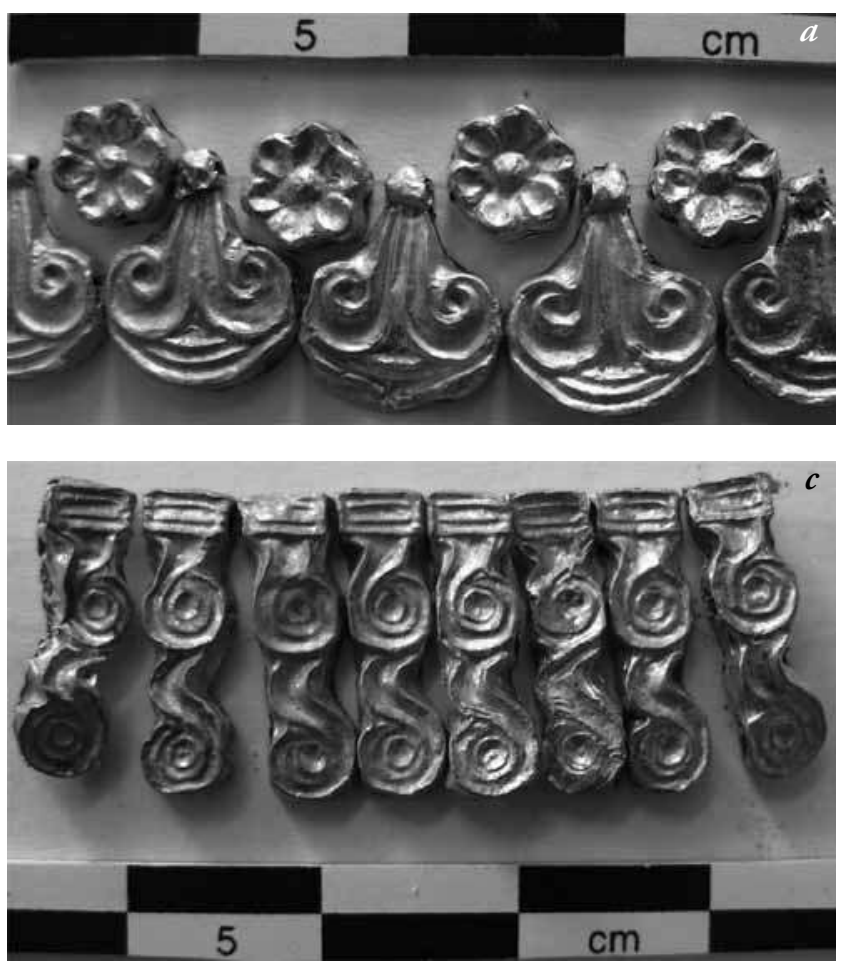

Les disques se séparent en trois groupes chimiques (Fig. 2), dont un s'approche de celui des feuilles. La couleur des feuilles se situe dans le vert-jaune du diagramme ternaire de couleur (Rapson, 1990), alors que la couleur des disques se situe dans la région jaune.

\section{Les colliers BE36137 et BE36161 et les perles BE36111}

Le collier BE36137 est constitué de perles creuses en forme de rosette à six pétales. Ces mêmes perles intercalées de perles en forme de fleur de lis constituent le collier $\mathrm{BE} 36161$. Les perles $\mathrm{BE} 36111$ sont à double spirale.

Toutes les perles sont fabriquées de façon identique : une feuille lisse à l'arrière (parfois pliée sur la feuille avant) et une feuille avant (Fig. 3a), dont le motif est obtenu par pression sur une matrice (Treister et Hargrave, 2001). Les perles sont percées de chaque côté (vers l'intérieur) d'un orifice pour BE36137 (environ $230 \mu \mathrm{m}$ de diamètre), de deux orifices pour BE36161 (environ $200 \mu \mathrm{m}$ de diamètre, Fig. 3b) et de trois orifices pour BE36111 (200-300 $\mu \mathrm{m}$ de diamètre). Malgré l'absence de soudures visibles sous binoculaire, il est possible que cette technique ait été utilisée (Schorsch, 1992). L'absence de traces d'usure indique que ces objets sont une production à but funéraire.

La composition de chaque collier est très homogène (Tableau 1). BE36161 est réalisé avec un alliage très proche

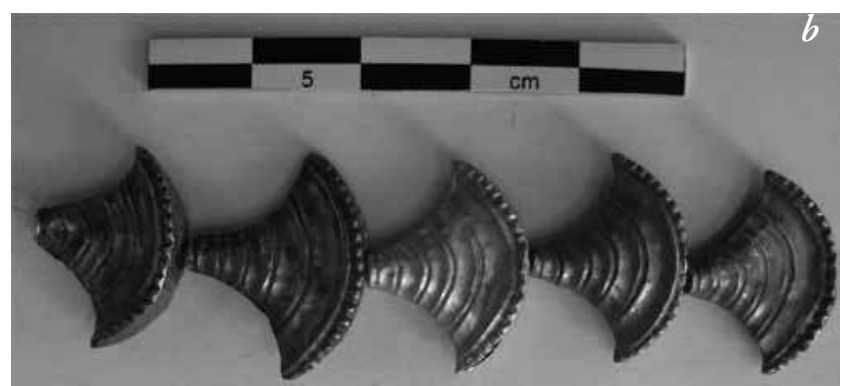

Figure 1 : (Voir planche couleur) Les perles : (a) en rosette et lis du collier BE36161; (b) à papyrus BE30000; (c) à double spirale BE36111.

Figure 1: (See colour plate) The beads: (a) rosette and lily from necklace BE36161; (b) papyrus BE30000; (c) double volute BE36111.

de celui des feuilles alors que BE36137 a une composition très proche de celle des disques de meilleure qualité. Malgré des teneurs en cuivre qui varient de 1,7 à $3,2 \%$, la teneur en argent de BE36111 est homogène mais inférieure aux teneurs mesurées pour les autres objets de Kazanaki. Les teneurs en argent et en cuivre des alliages se situent dans les valeurs espérées pour l'or natif et leurs couleurs se partagent entre les régions vert-jaune et jaune du diagramme ternaire de couleur (Rapson, 1990).

\section{Les perles BE30000}

Cinq perles creuses à papyrus BE30000 (motif utilisé à l'époque minoenne en orfevvrerie et céramique, Evans, 1921), présentant des signes d'usure, sont de couleur, d'épaisseur de feuille et de facture distinctes des autres perles étudiées dans ce travail. Deux feuilles estampées sont soudées pour former la perle, qui est percée en haut et en bas, vers l'intérieur, d'orifices de $300 \mu \mathrm{m}$ de diamètre. Les alliages possèdent des teneurs en argent de 15 à $21 \%$ et en cuivre de 2 à $9 \%$. Leur couleur va du jaune rougeâtre au jaune verdâtre du diagramme ternaire de couleur (Rapson, 1990).

\section{Les alliages et l'origine de l'or}

La concentration d'or est inférieure à $89 \%$ pour tous les objets analysés, ceci concordant avec la composition des feuilles de la tombe 4 de Mycènes (Schliemann, 1878), d'une coupe mycénienne dite de Syrie (Hackens, 1983), de deux objets mycéniens publiés par Hartmann en 1970, de feuilles et globules de creuset trouvés dans une tombe mycénienne en Thessalie et des objets de Kastanas (Vavelidis et Andreou, 2007) (voir Tableau 2). 


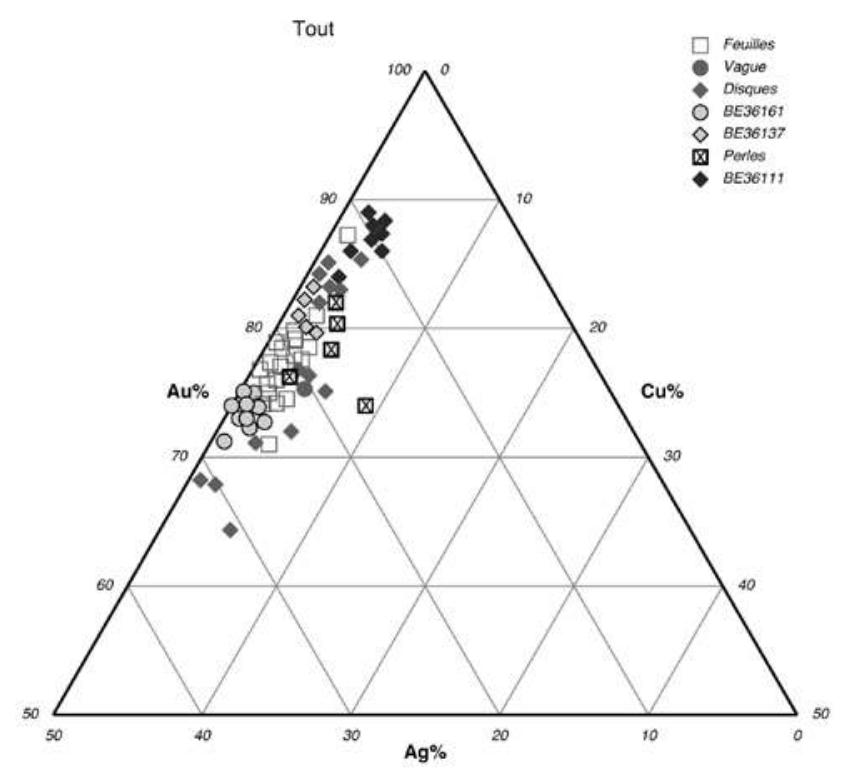

Figure 2 : Diagramme ternaire représentant les éléments $\mathrm{Au}-\mathrm{Ag}-\mathrm{Cu}$ (en \%) pour les objets de la tombe de Kazanaki analysés par PIXE et par FX portable.

Figure 2: Ternary diagram for the base-alloys of the objects from Kazanaki, Au-Ag-Cu (in \%), analysed by PIXE and by portable XRF.

Les deux colliers et les perles à double spirale possèdent une composition élémentaire très homogène pour chaque ensemble, ce qui permet de suggérer une fabrication unique par ensemble. Si l'on en exclue deux, les feuilles d'or possèdent aussi une composition homogène, contrairement aux disques (l'analyse d'un plus grand nombre pourrait révéler trois groupes distincts) et aux cinq perles à papyrus.

Tous les alliages contiennent des teneurs en argent typiques des ors alluvionnaires (Raub, 1995), néanmoins les teneurs en cuivre de certains de ces objets ne peuvent être obtenues que par ajout volontaire (Guerra et Rehren, 2009; Hauptamann et Klein, 2009). La teneur en cuivre de certains disques et de trois perles à papyrus peut atteindre, respectivement, 6 et $9 \%$. Rappelons que la cémentation est un procédé métallurgique qui n’apparaît qu'au $\mathrm{I}^{\mathrm{er}}$ millénaire av. J.-C. (Ramage et Craddock, 2000); la couleur et les propriétés des alliages à l'époque mycénienne dépendent alors de la teneur en argent des ors disponibles et ne peuvent être modifiées que par ajout de cuivre. Les concentrations en cuivre mesurées pour nos objets varient entre 0,5 et $9 \%$. Les concentrations mesurées par Vavelidis et Andreou (2007) pour des pépites provenant de gisements alluvionnaires en Grèce, pour Kastanas et pour les feuilles d'or ne dépassent pas $0,5 \%$ (alors que l'argent varie de 1 à $22 \%$ ); des valeurs identiques ont été mesurées pour les objets mycéniens publiés par Schliemann (1878), Hartmann (1970);
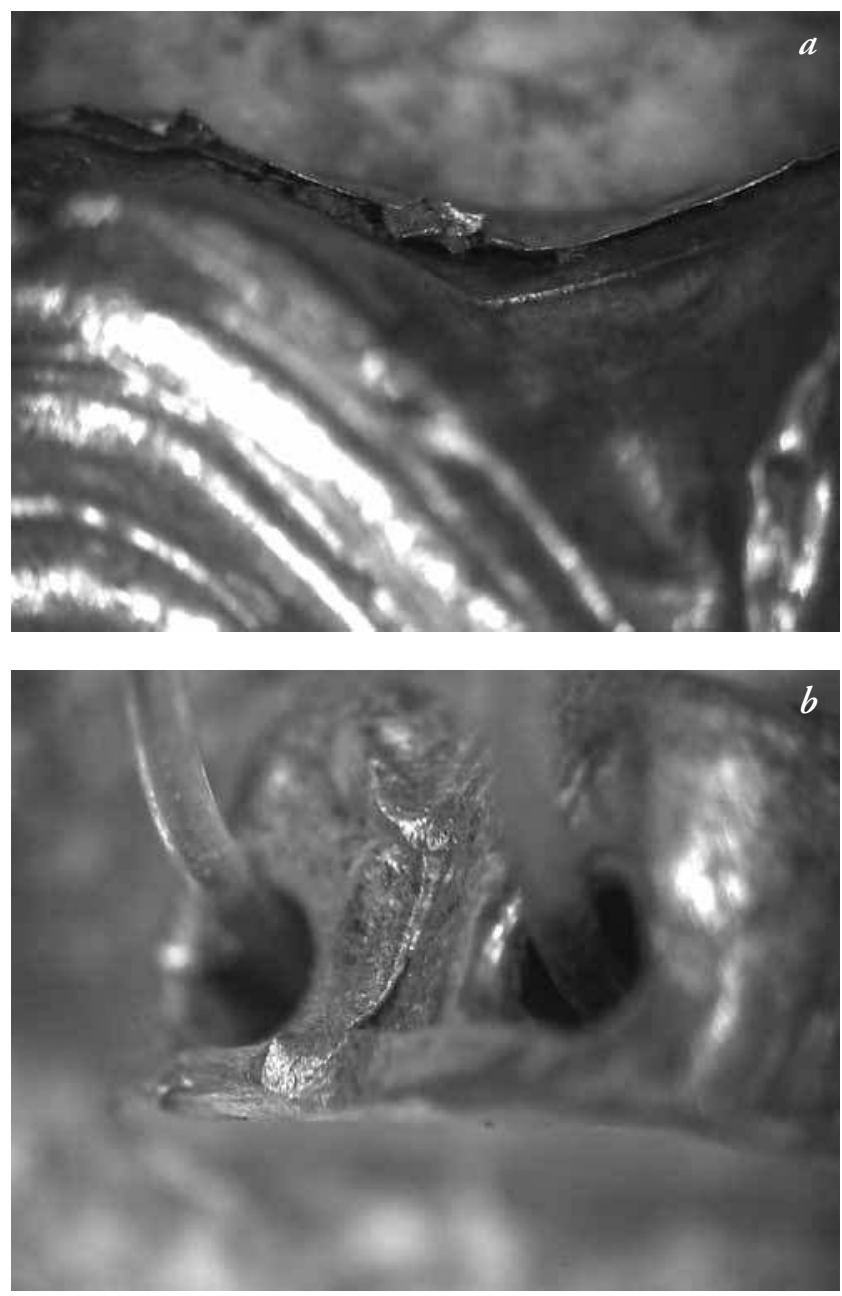

Figure 3 : Détails sous loupe binoculaire du montage des perles à : (a) double spirale BE36111; (b) rosette et lis du collier BE36161. Figure 3: Details under the stereomicroscope of the mounting of: (a) double volutes beads BE36111; (b) rosette and lily beads from necklaces BE36161.

Hackens, 1983. Néanmoins, le globule de creuset publié par Vavelidis et Andreou, 2007 possède 3,5 \% de cuivre. L'addition de cuivre à des ors alluvionnaires est une pratique qui semble avoir été employée aussi à la même époque en Égypte et en Géorgie (respectivement Troalen et al., 2009; Hauptmann et Klein, 2009) et en Anatolie dès le milieu du III ${ }^{e}$ millénaire (Swann et al., 1997).

En ce qui concerne la question de l'origine de l'or, l'analyse de 17 feuilles et disques par PIXE montre des teneurs en Sn qui peuvent atteindre $850 \mathrm{ppm}$ (Tableau 2). Un groupe chimique avec de fortes teneurs en Pd et $\mathrm{Sn}$ est accompagné d'une droite (Fig. 4) où s'alignent les autres objets, notamment les trois disques analysés. La présence de $\mathrm{Sn}$ et/ou Pt dans les ors est en général liée à l'exploitation de gisements 


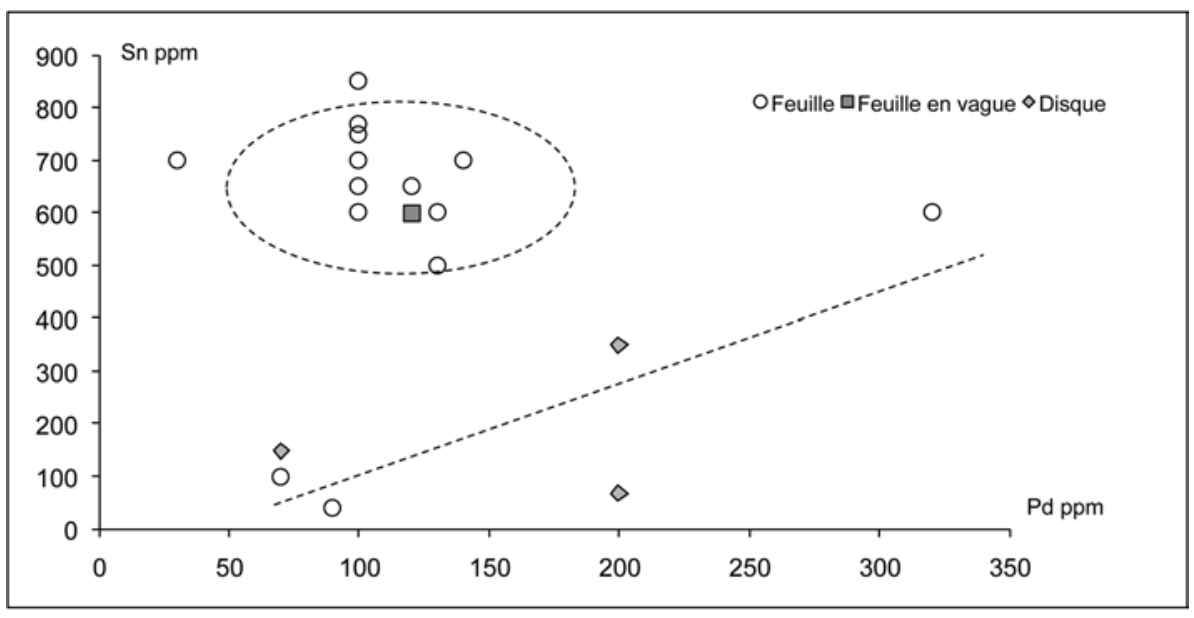

Figure 4 : Représentation des teneurs en Pd et en $\mathrm{Sn}$ (en ppm) mesurées par PIXE.

Figure 4: Pd and Sn contents (in ppm) measured by PIXE. alluvionnaires (Dube, 2006). Néanmoins, aucune inclusion de platinoïdes (Meeks et Tite, 1980) n’a pu être décelée sur la surface des objets. Malgré la taille et l'épaisseur des feuilles d'or, nous avons estimé par PIXE-XRF les concentrations de Pt pour six d'entre elles. Les résultats montrent qu'une partie des objets possède des teneurs inférieures à la limite de détection $(80 \mathrm{ppm})$ alors que les autres possèdent des concentrations estimées à 200-500 ppm. Seul un plus grand nombre d'analyses permettrait de vérifier l'existence de deux groupes.

La présence de Sn et de Pt dans les alliages conforte l'hypothèse de l'utilisation d'ors alluvionnaires à Volos pendant la période mycénienne. Néanmoins, le manque de données sur les caractéristiques géochimiques des sources d'or à l'Age du Bronze en Méditerranée, en Égypte et dans le Levant - nous rappelons les circuits commerciaux à cette époque, notamment le lapis-lazuli qui devrait provenir d'Afghanistan (Hughes-Brock, 1999) - nous empêche de proposer à présent une provenance pour ces ors. Il faudra néanmoins remarquer que les ors analysés par Hauptmann et Klein (2009) de mines en Géorgie exploitées à l'Âge du Bronze présentent de faibles teneurs en $\mathrm{Sn}$ et $\mathrm{Pt}$.

\section{Conclusion}

L'étude analytique de feuilles, disques et perles de colliers de la tombe à tholos de Kazanaki nous a permis de réaliser une première approche aux pratiques orfevres et à l'origine de l'or en Thessalie à l'époque mycénienne. Si l'on excepte les perles à papyrus, tous les objets ont vraisemblablement été produits dans un but funéraire, à partir de fines feuilles d'or, parfois décorées par estampage. Les perles sont creuses, obtenues par assemblage de deux coques et ensuite percées.
Les disques et les feuilles sont percés pour application sur des vêtements.

Les teneurs en argent varient entre 9 et $31 \%$ et en cuivre entre 0,5 et $9 \%$. Si les teneurs en argent sont typiques des ors natifs, les teneurs en cuivre dépassant $2 \%$ correspondent à des alliages volontaires. Nous pouvons suggérer la recherche d'effets polychromes par simple ajout de cuivre à des ors natifs, dont la quantité d'argent est variable, ce qui laisse un large choix à l'orfèvre. Les ensembles sont homogènes, sauf les perles à papyrus et les disques. L'analyse de la totalité des disques pourrait montrer trois groupes homogènes correspondant à trois séries de fabrication distincte. L'homogénéité des alliages pourrait, après analyse de tous les objets de la tombe, séparer les productions par enterrement.

L'analyse PIXE et PIXE-XRF de feuilles et disques révèle la présence de $\mathrm{Sn}$ et $\mathrm{Pt}$, éléments caractéristiques des ors alluvionnaires. Les ors mycéniens de Volos peuvent ainsi avoir été exploités grâce à l'utilisation de peaux de béliers, ou toisons d'or. Si l'origine alluvionnaire de ces ors est clairement prouvée, leur provenance reste non identifiée par manque de données géochimiques. Seule l'analyse d'objets provenant d'autres sites mycéniens et de sites en Géorgie chronologiquement proches, permettrait de montrer le rapport entre la Thessalie et la Colchide prouvant que les ors de Kazanaki sont ceux de la mythique Toison d'or.

\section{Bibliographie}

Adrimi-Sismani, V., 2004-2005. Le palais de Iolkos et sa destruction, $B C H$ 128-129, 1. Études, 1-54.

Adrimi-Sismani, V., 2007. Iolkos: Myth, Archaeology and History, in Gordeziani R. et al. (eds), The Argonautica and World Culture (Phasis, Greek and Roman Studies 10,1), Tbilisi: 20-32. 
Adrymi-Sismani, V., 2008. The Mycaenean tholos tomb at Kazanaki, Annual Report of the British School at Athens : 59-61, pl. 103-105.

Adrimi-Sismani, V. et Godart, L., 2005. Les inscriptions en Linéaire B de Dimini/Iolkos et leur contexte archéologique, Annuario della Scuola Archeologica di Atene LXXXIII, Serie III, 5- Tomo I: 47-69.

DubE, R.K., 2006. Interrelation between gold and tin: a historical perspective, Gold Bulletin 39(3) : 103-113

Evans, A. 1921. The Palace of Minos at Knossos, vol. 1 : The Neolithic and Early and Middle Minoan ages, London, MacMillan and Co. Limited.

Gianoncelli, A., Castaing, J., Bouquillon, A., Polvorinos, A., et WAlter, P., 2006. Quantitative elemental analysis of Della Robbia glazes with a portable XRF spectrometer and its comparison to PIXE methods, X-ray spectrometry 35(6) : 365-369.

Guerra, M. F., Calligaro, T., Dran, J.-C., Moulherat, C. et SAlomon, J., 2005. Development of a PIXE, PIGE and PIXEXRF combination for the analysis of the gold from the First Empire of the Steppes, Geoarcheological and Bioarchaeological Studies 3 : 343-346.

Guerra, M.F. 2004. Fingerprinting ancient gold with proton beams of different energy, Nucl. Instrum. and Methods B 226 : 185-198.

Guerra, M. F. et Calligaro, T., 2004. Gold traces to trace gold, Journal of Archaeological Sciences 31, 1199-1208.

Guerra, M. F. et Rehren, Th., 2009. In-situ examination and analysis of the gold jewellery from the Phoenician tomb of Kition (Cyprus), ArchéoSciences 33.

Hackens, T., 1983. Gold Jewellery: craft, style \& meaning from Mycenae to Constantinopolis, Louvain-la Neuve, BelgiumArt and Archaeology Publications.

Hartmann, A., 1970. Prähistorische Goldfunde aus Europa, Band 2: Spektralnalytische Untersuchungen und deren Auswertung, Berlin, Gebr. Mann Verlag.

Hauptmann, A. et Klein, S., 2009. Bronze Age gold in southern Georgia, ArchéoSciences 33.

Higgins, R.A., 1980. Greek and Roman jewellery, $2^{\text {nd }}$ edition, University of California Press, Berkeley and Los Angeles.

Hughes-Brock, H., 1999. Mycenaean beads: Gender and social contexts, Oxford journal of archaeology 18(3) : 277-296.

Meeks, N.D. et Tite, M.S., 1980. The analysis of Platinumgroup Element inclusions in Gold Antiquities. Journal of Archaeological Science 7: 267-275.
Papanastasiou, A., 2006. To osteologiko yliko apo to tholoto tapho ste thesi Kasanaki, Proceedings of the $2^{\text {nd }}$ Conference "Archaeologiko Ergo Thessalias kai Stereas Ellados", Volos-University of Thessaly, 151-161.

Ramage, A. et Craddock, P.T., 2000. King Croesus' gold: Excavations at Sardis and the History of Gold Refining. London, British Museum Press.

Raub, Ch. J., 1995. The metallurgy of gold and silver in prehistoric times, in Prehistoric Gold in Europe, G. Morteani \& J. P. Northover eds, 243-259.

Rapson, W. S., 1990. The metallurgy of the coloured carat gold alloys. Gold Bull. 23(4) : 125-133.

Schliemann H., 1878. Mykenae und Tiryns, Leipzig, F. A. Brockhaus.

Schorsch, D., 1995. The gold and silver necklaces of Wah: a technical Study of an unusual metallurgical joining method, in Conservation in ancient Egyptian collections, Brown C., Macalister F., Wright M. (editors), Archetype Publications, London: 127-135.

Solé, V. A., Papillon, E., Cotte, M., Walter et P., Susini, J., 2007. A multiplatform code for the analysis of energy-dispersive X-ray fluorescence spectra, Spectrochimica Acta Part B-Atomic Spectroscopy 62(1) : 63-68.

Swann, C.P., Betancourt, P.P., Fleming, S. et Floyd, C.R., 1997. PIXE analysis of Trojan gold jewelry, Nucl. Instrum. and Methods $\mathrm{B} 130$ : 320-323.

Troalen, L., Guerra, M.F., Tate, J., Manley, W.P., 2009. Technological study of gold jewellery pieces dated from Middle Kingdom to New Kingdom in Egypt. ArchéoSciences 33.

Vavelidis, M. et Andreou, S., 2008. Gold and gold working in Late Bronze Age Northern Greece, Naturwissenschaften 95 : 361-366.

Treister, M Y. et Hargrave J., 2001. Hammering Techniques in Greek and Roman Jewellery and Toreutics, Colloquia Pontica 8, Brill Academic Publishers, Leiden.

Viguerie, L. de, Duran, A., Bouquillon, A., Solé, V. A., Castaing, J. et Walter, P., 2009. Quantitative X-ray fluorescence analysis of an Egyptian faience pendant and comparison with PIXE, Anal Bioanal Chem, DOI 10.1007/s00216-0092974-7.

Zehnacker, H., 1983 (traduction). Pline l'Ancien, Histoire Naturelle, livre XXXIII, Paris, Belles Lettres. 
